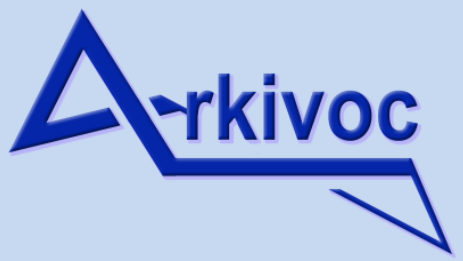

Free to Authors and Readers
A Platinum Open Access Journal for Organic Chemistry

Paper

DOAJ Seal
Arkivoc 2021, part iv, 105-112

\title{
Striking difference in reactivity of trienes and oximes derived from glucose and xylose
}

\author{
Jarosław Jaźwinski, ${ }^{a}$ Paweł Łata, ${ }^{\mathrm{b}}$ Karolina Tiara, ${ }^{\mathrm{a}}$ and Sławomir Jarosz ${ }^{\mathrm{a} *}$ \\ a Institute of Organic Chemistry, Polish Academy of Sciences, Kasprzaka 44/52, \\ 01-224 Warsaw, Poland \\ ${ }^{b}$ Institute of Physical Chemistry, Polish Academy of Sciences, Kasprzaka 44/52, \\ 01-224 Warsaw, Poland \\ Email: slawomir.jarosz@icho.edu.pl
}

This paper is dedicated to Professor Horst Kunz on the occasion of his 80th anniversary

Received 09-18-2020

Accepted 10-09-2020

Published on line 10-21-2020

\section{Abstract}

D-Glucose was converted into a dieno-oxime and independently into a triene. Both derivatives were completely unreactive and did not undergo any cyclization: either [1,3]-dipolar cycloaddition (oxime) or [4+2] cycloaddition (triene). This result was very surprising comparing to similar examples observed by us for derivatives of 'shorter' sugar: D-xylose. The big difference in reactivity of the pentose and hexose derivatives is clarified by an in silico study.
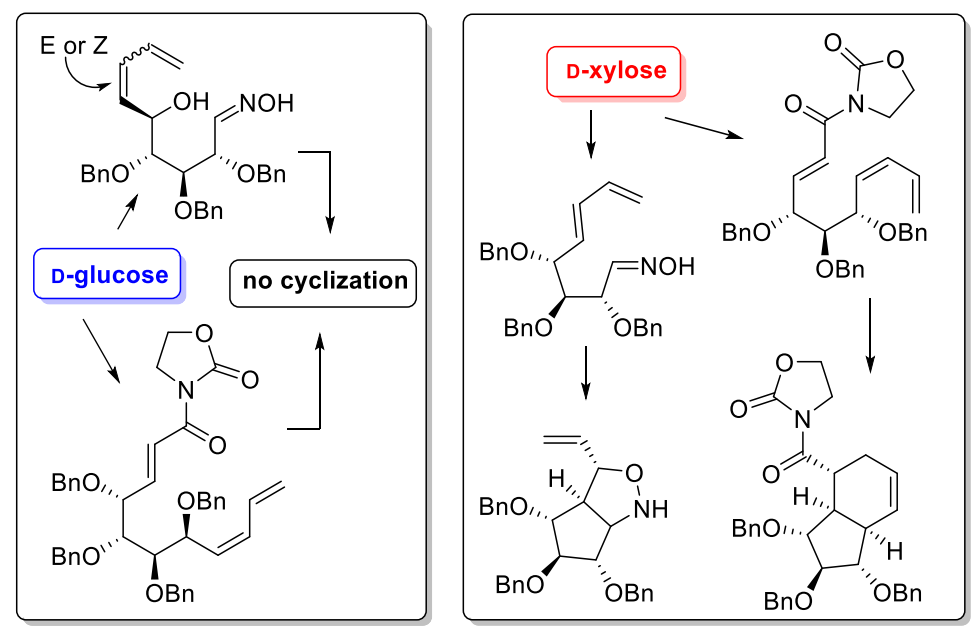

Keywords: cyclization, sugar oximes, sugar trienes, molecular orbitals, DFT calculations. 


\section{Introduction}

Elaboration of convenient synthetic routes to bicyclic sugar mimetics is one of the leading trends in our laboratory. ${ }^{1,2}$ We have proposed an efficient method for the stereoselective preparation of sugar dienes with the $E$ or $Z$ geometry across the internal double bond from $D$ glucose (1-E or $\mathbf{1 - Z}$; Scheme 1$).^{3}$

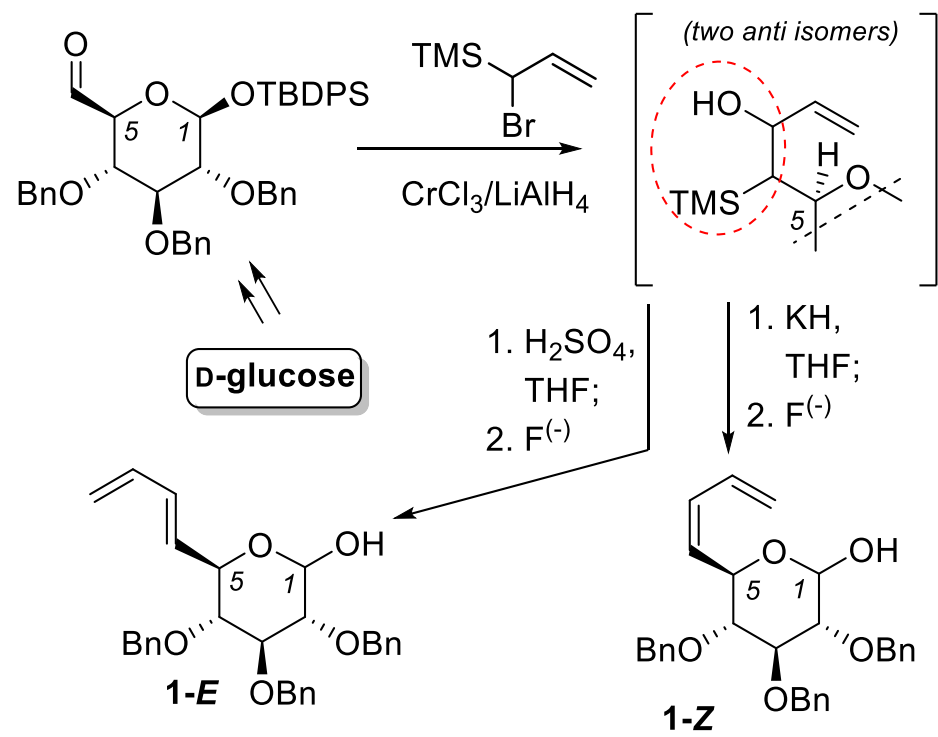

Scheme 1. Effective synthesis of sugar dienes with the precisely defined configuration across the internal double bond (ref. 3).

These derivatives could eventually be used as important intermediates in the synthesis of a variety of sugar mimetics as shown in Figure 1. Proper functionalization at the anomeric center in both dienes should provide either oximes $\mathbf{2}$ or trienes 3; cyclization of them would afford the corresponding bicyclic derivatives $\mathbf{4}$ (which could be converted into 5) and 6 (Figure 1).

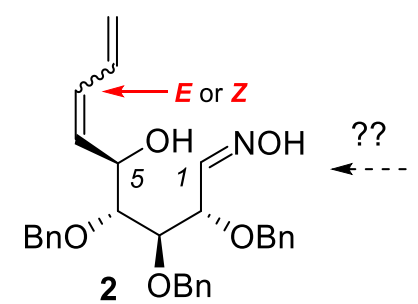

1,3-dipolar $\mid$ cycloaddition

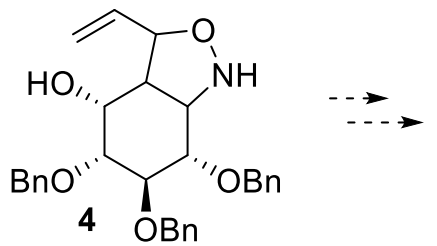

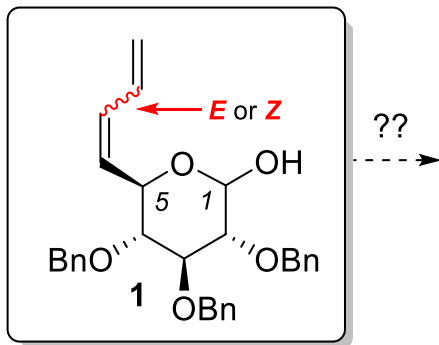

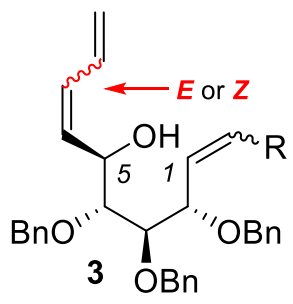

$[4+2] \mid \begin{aligned} & ? ? \\ & \text { cycloaddition }\end{aligned}$<smiles>OC1C=CCNC2C1[C@H](O)[C@H](Br)[C@H](OCc1ccccc1)[C@H]2OCc1ccccc1</smiles><smiles>[R]C1CC=CC2C1[C@H](O)[C@H](O)[C@H](O)[C@H]2OCc1ccccc1</smiles>

Figure 1. Proposed routes to useful intermediates for bicyclic sugar mimics. 
This assumption was based on our previous results describing the successful cyclization of the corresponding oxime 7 derived from xylose, and triene $\mathbf{8 ,}^{1,4,5}$ allowing the stereoselective preparation of bicyclic carba- and imino-sugars ( 9 and 10 respectively) in good yield (Figure 2). Further support for this hypothesis came also from the results of Evans who was able to prepare derivative of decalin 12 by a stereoselective cyclization of triene $\mathbf{1 1 .} .^{6-8}$

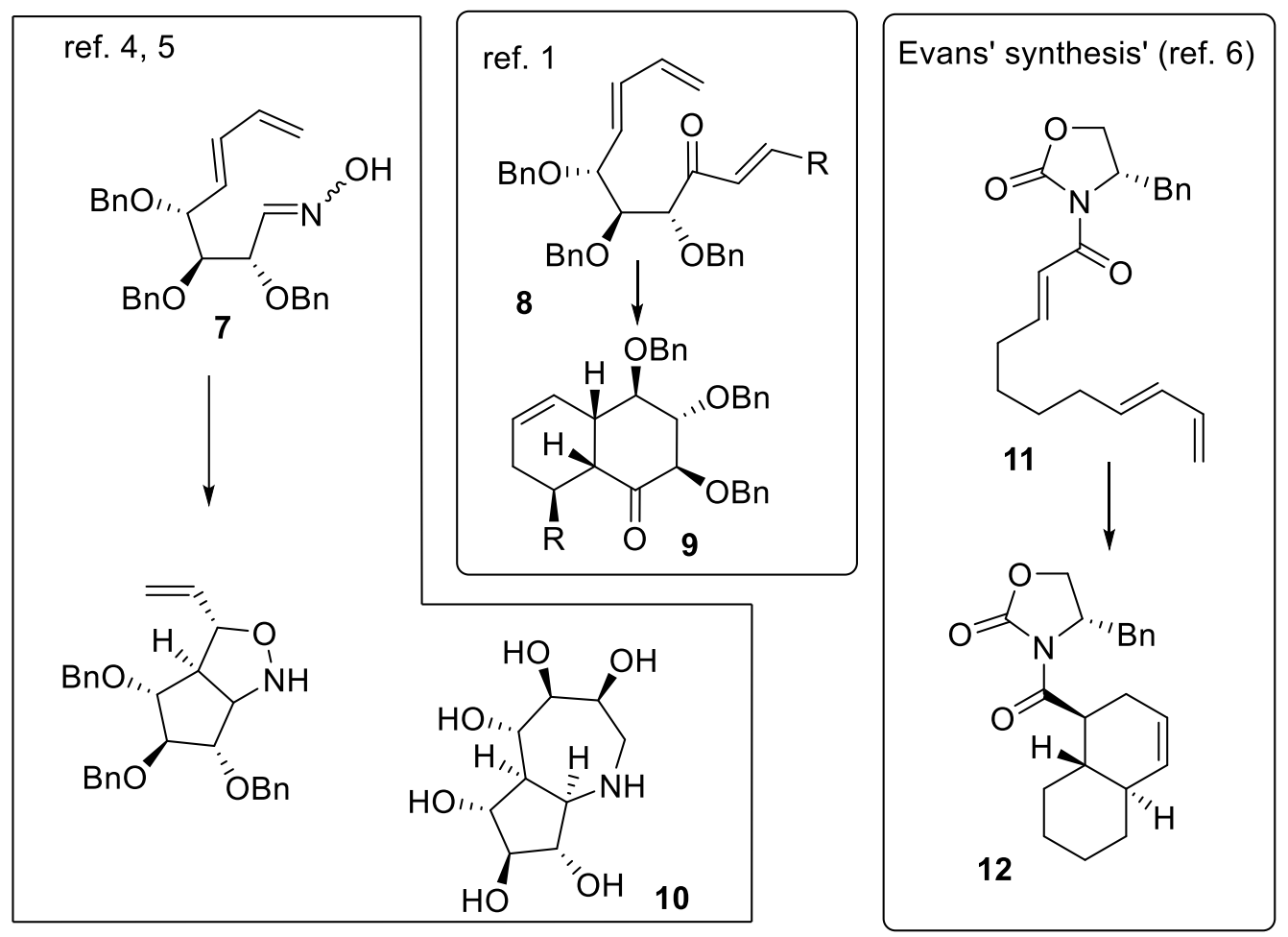

Figure 2. Synthesis of bicyclic sugar mimetics involving pericyclic reactions as key-steps.

\section{Results and Discussion}

We have succeeded to introduce the oxime function at the $\mathrm{C}-\mathbf{1}$ in both dienes (1-E and $\mathbf{1 - Z})$; $^{3}$ the resulting oximes $\mathbf{2}-\boldsymbol{E}$ and $\mathbf{2}-\mathbf{Z}$ should undergo the [1,3]-dipolar cycloaddition (in this case called also an oxime/olefin cyclization $^{9}$ ) as reported for a 'shorter' oxime 7 (cf. Figure 2). However, both oximes were very resistant towards such process ( $2 E$ or $\mathbf{2 Z} \rightarrow \mathbf{4}$; see Fig. 1); we were not able to induce this cyclization even under very high pressure (10 000 bar).

Synthesis of trienes 3 by reaction of acetals $\mathbf{1 - E}$ and $\mathbf{1 - Z}$ with Wittig reagents also failed. These acetals were very resistant also towards even simplest organometallic reagents such as e.g. Grignard reagents.

Recently we have succeeded in the preparation of the 'inverted' to $\mathbf{3}$ trienes, i.e. compounds in which the diene function is located at the anomeric center of glucose and the olefinic part at the terminal position (13 in Figure 3). However, such trienes also did not undergo any cyclization, which should afford the bicyclic derivative 15. ${ }^{10}$ This fact is very odd, since similar triene $\mathbf{1 4}$ - derived from a shorter analog (D-xylose) - was readily converted into bicyclic derivative 16 upon treatment with a Lewis acid. Also, the analog of 13 
containing no alkoxyl groups underwent smooth cyclization to decalin derivative $\mathbf{1 2}$ as already reported by Evans $^{6-8}$ (see Figure 2).
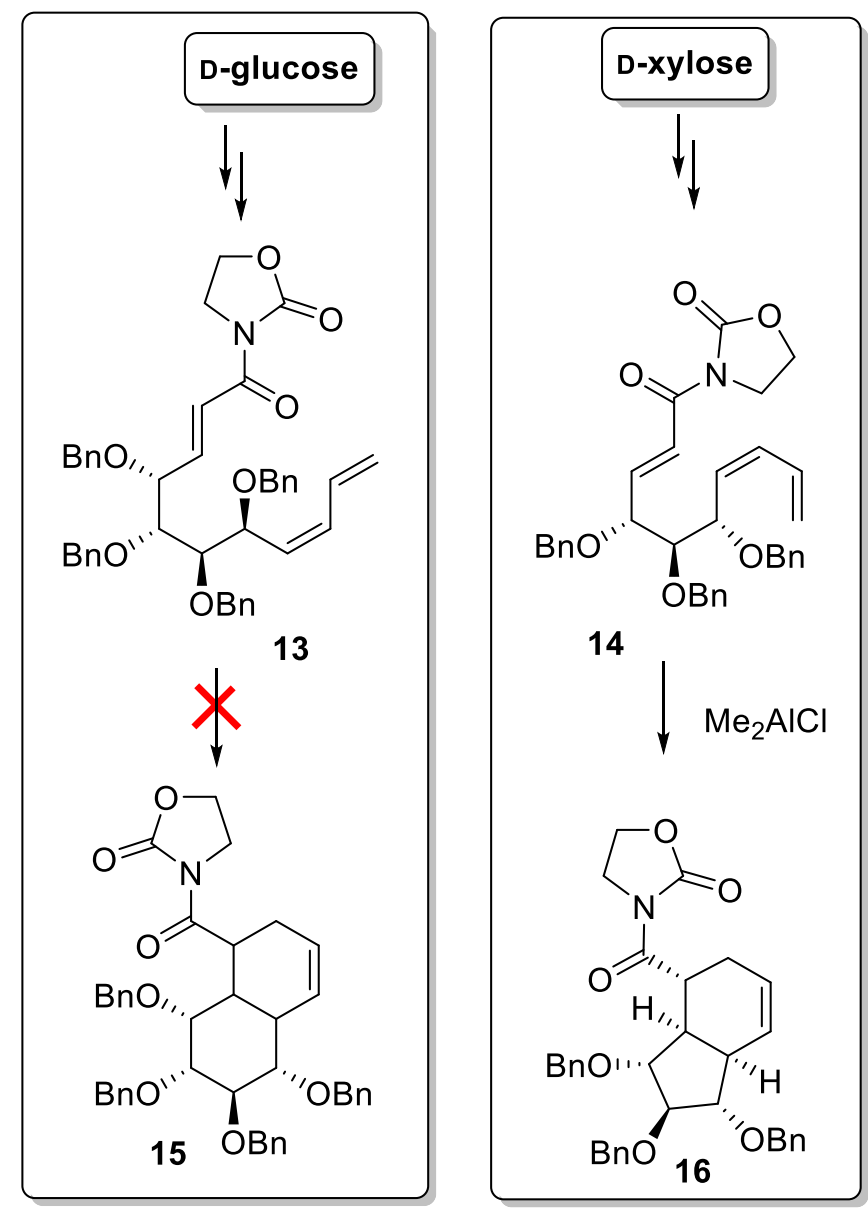

Figure 3. Cyclization of the D-xylose derived triene $\mathbf{1 4}^{\mathbf{1 0}}$ and attempts to cyclize D-glucose derived triene 13 .

The unusual resistance of the triene and oximes derived from glucose (compounds 13 and $2 E / \mathbf{2 Z}$ respectively) to cyclization is also explained by in silico approach.

\section{$\boldsymbol{A b}$ initio calculations of the cyclization reactions}

All calculations were performed using the Gaussian 09.D.01 package. ${ }^{11}$ Calculations of molecular orbitals (MO) were at the B3LYP/6-311G(2d,p) theory level, using B3LYP/6-31G(2d,p) optimized compound geometries. As input, arbitrarily selected conformers were used. All calculations were performed for isolated molecules in gas phase. We explain the reactivity by analysis of the Frontier Molecular Orbitals (FMO) of reagents.

\section{Reactivity of oximes}

The molecular orbitals of both compounds $\mathbf{2}$ and $\mathbf{7}$ show similar features, namely, HOMO and LUMO orbitals are localized on the diene fragments. Neither HOMO nor LUMO orbitals are localized on the $\mathrm{C}=\mathrm{C}$ bonded to oxime group. In contrast, occupied and non-occupied orbitals involving the $\mathrm{C}=\mathrm{N}-\mathrm{OH}$ group are spread over the entire molecule, mainly on the phenyl rings. The difference in reactivity of $\mathbf{2}$ and $\mathbf{7}$ can be explained by a degree of delocalization of these orbitals (Figures 4 and 5). 


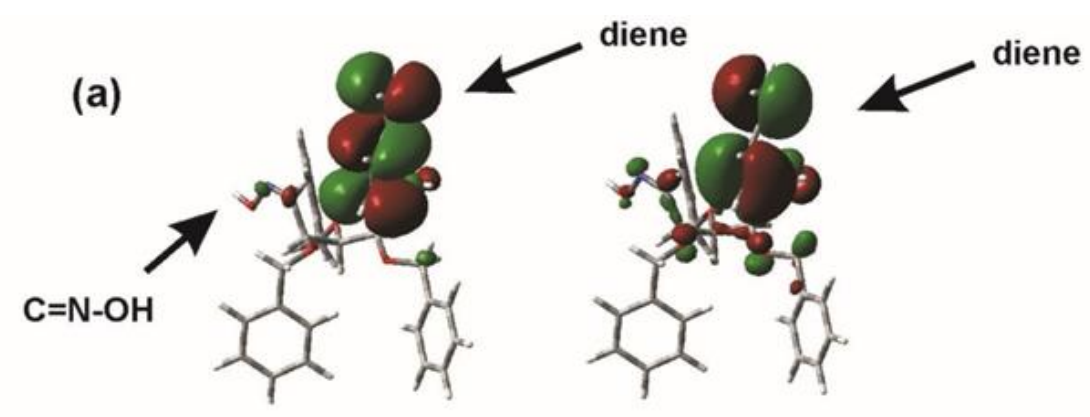

(b)
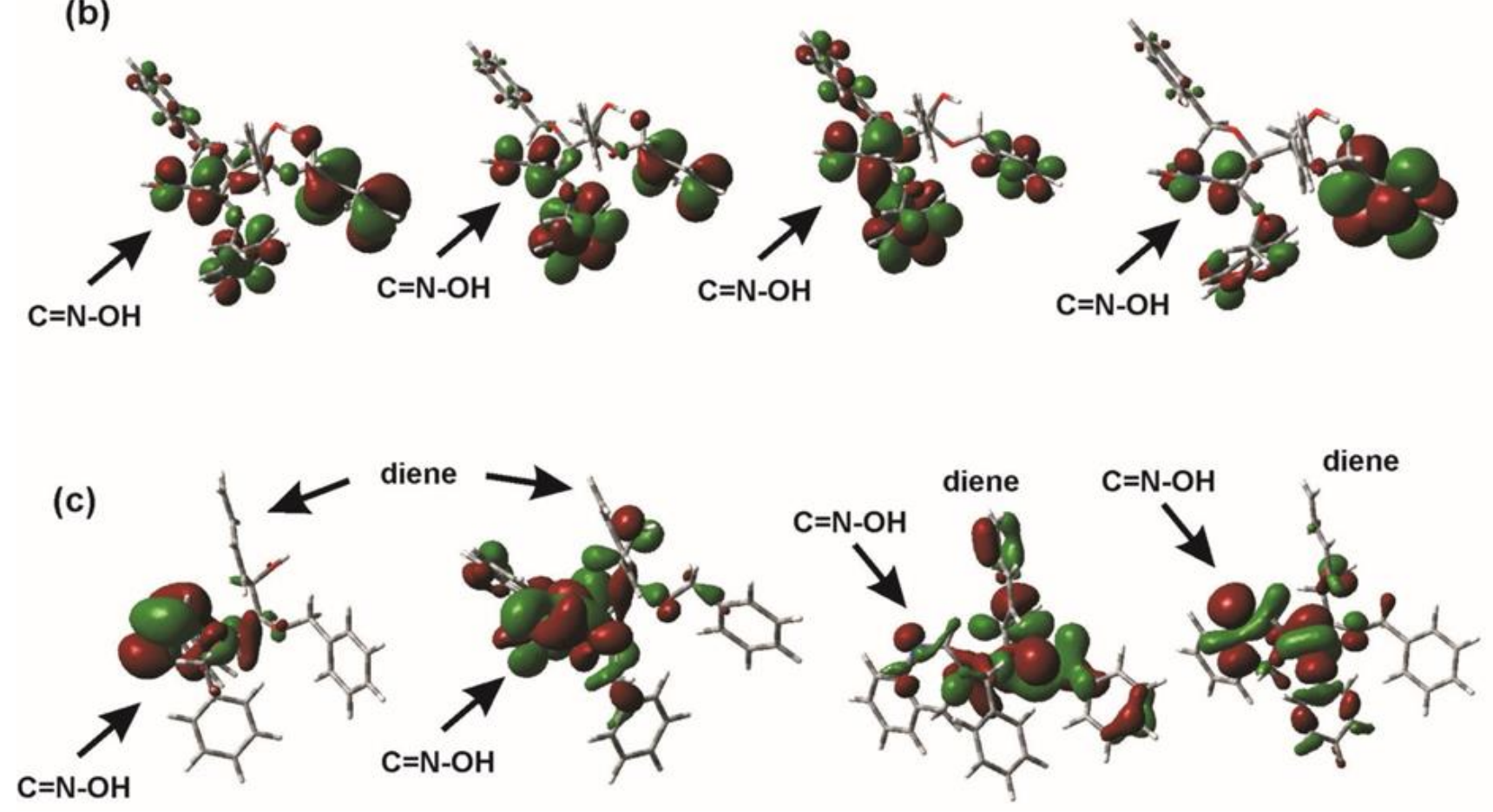

Figure 4. Selected molecular orbitals of 2. (a) LUMO (left) and HOMO. Both orbitals are localized on the diene fragment. (b) LUMO+1, LUMO+2, LUMO+3, and LUMO+4. All these orbitals are shared between $\mathrm{C}=\mathrm{N}-\mathrm{OH}$ and phenyl groups. (c) HOMO-9, HOMO-10, HOMO-11, and HOMO-12; all orbitals involve the center of reactivity of the molecule. The remaining orbitals, from HOMO-1 to HOMO-8 are localized on the phenyl groups and do not involve the $\mathrm{C}=\mathrm{N}-\mathrm{OH}$ fragment. 

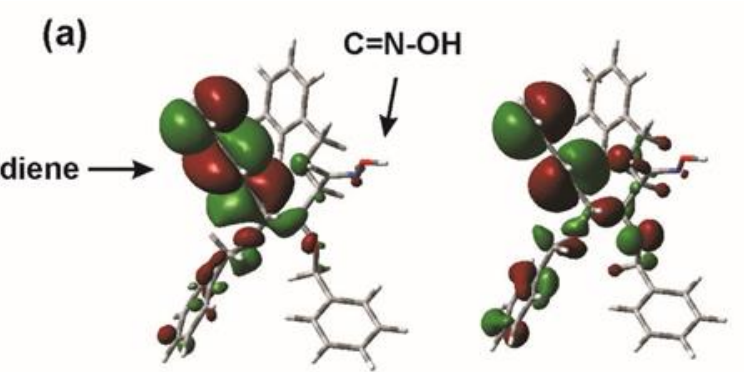

(c)

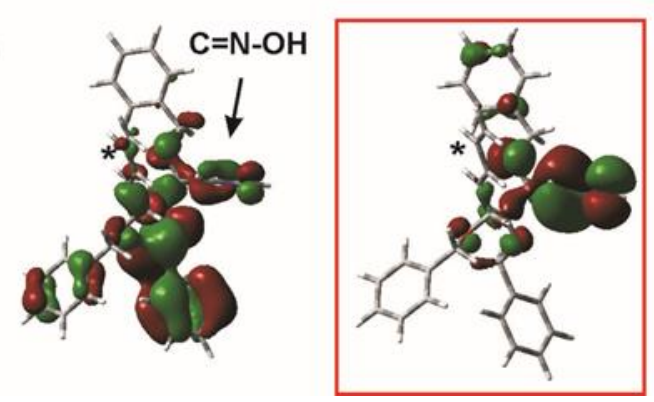

(b)
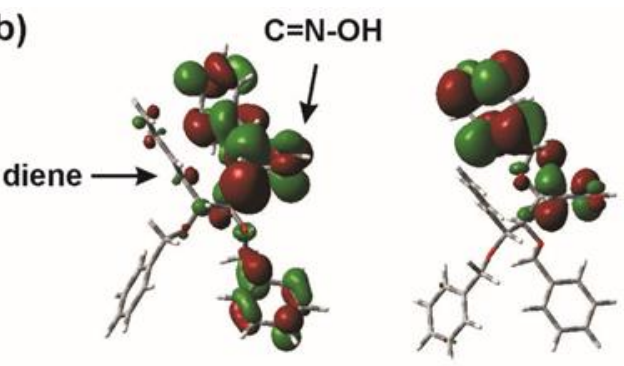

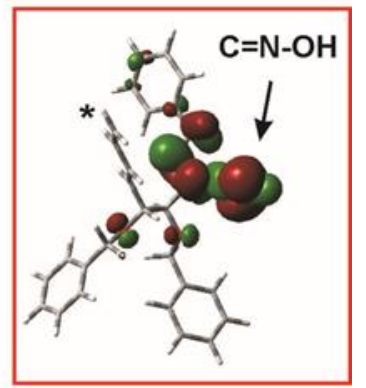

Figure 5. Selected molecular orbitals of 7. Asterisks indicate the diene fragment. (a) LUMO (left) and HOMO (right). Both orbitals are localized at the diene fragment. (b) LUMO+1 and LUMO+2. Orbitals are spread over the atoms of $\mathrm{C}=\mathrm{N}-\mathrm{OH}$ and phenyl groups. (c) HOMO-7, HOMO-8, HOMO-10 and HOMO-11 involving the oxime fragment. The (LUMO) - (HOMO-8) gap amounts to $6.2 \mathrm{eV}$. Two unoccupied and two occupied orbitals (red frames) are localized on the $\mathrm{C}=\mathrm{N}-\mathrm{OH}$ fragment.

\section{Reactivity of the trienes}

The Diels-Alder (DA) reactions can be explained by the topology of FMOs, by the interaction of the HOMO orbital of the diene and LUMO orbital of the dienophile. This is the case of trienes 11 and 14 . The HOMO and LUMO orbitals are localized on the diene and dienophile components, and the DA reaction can occur. In contrast, for 13 the HOMO orbital is localized on the phenyl group, whereas only HOMO-1 orbital is localized on the diene moiety. The lack of HOMO-LUMO interaction makes the DA reaction difficult (Figure 6). 
(a)

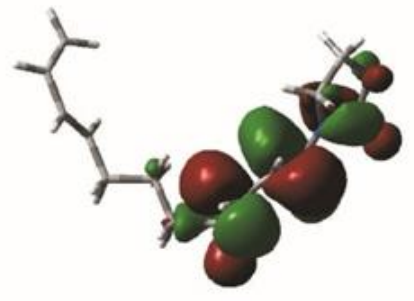

(b)

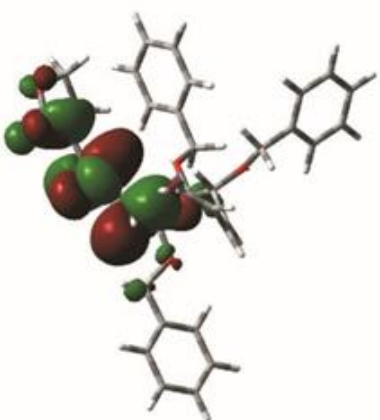

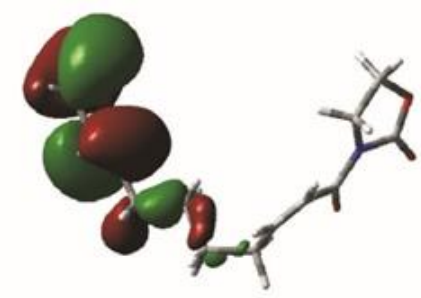

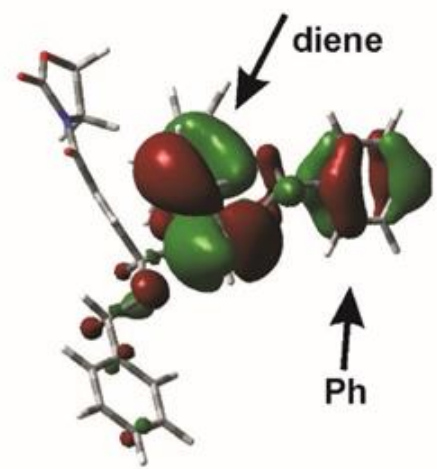

(c)

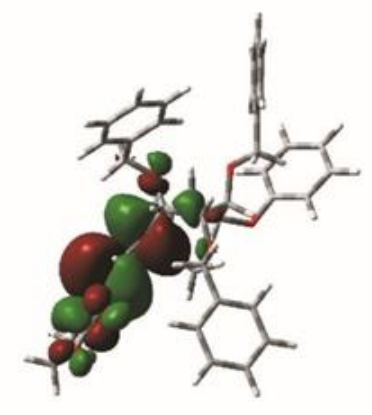

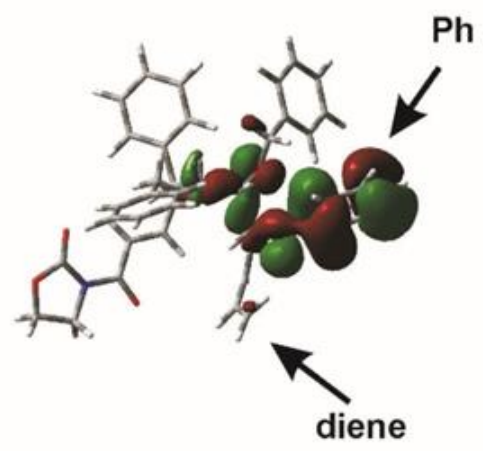

$\mathrm{Ph}$

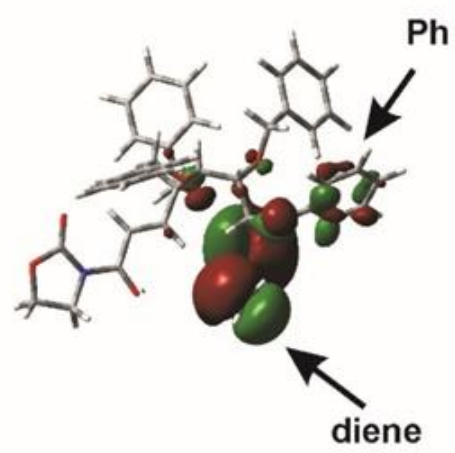

Figure 6. (a) LUMO (left) and HOMO (right) molecular orbitals of 11. LUMO-HOMO gap amounts to $4.7 \mathrm{eV}$ (b) LUMO (left) and HOMO (right) of 14. HOMO orbital is spread over atoms of the diene and phenyl groups. LUMO-HOMO gap amounts to $4.8 \mathrm{eV}$. (c) LUMO (left), HOMO (middle) and HOMO-1 orbitals of 13. The HOMO orbital is localized at the phenyl group.

\section{Conclusions}

We have demonstrated that great care should be taken analyzing pericyclic reactions carried out for very similar substrates. The triene derived from D-xylose (having three benzyloxy groups in the chain) underwent readily [4+2]-cyclization affording the bicyclic product in high yield and high stereoselectivity. However, very similar but 'longer' triene derived from D-glucose (with four OBn groups in the chain) was completely unreactive under the same conditions. Interestingly, the longer triene (the same length as that from glucose) but without any alkoxyl groups underwent facile [4+2] reaction. This strange behavior can be explained by the interaction of frontier orbitals which play a vital role in pericyclic reactions.

The same situation was observed by us in the cyclization of sugar dieno-oximes. Again, the 'shorter' oxime (derived from xylose) underwent facile 1,3-dipolar cycloaddition, while longer (derived from glucose) was completely unreactive. This phenomenon was also explained by the interaction of appropriate orbitals. 


\section{Experimental Section}

Synthetic work for the compounds described in this paper is reported elsewhere. ${ }^{1,3-5,9,10}$

\section{Supplementary Material}

Calculated molecular orbitals of compounds 2, 7, 11 (two conformers), 13 and 14.

\section{References}

1. Review: Jarosz, S. Curr. Org. Chem., 2008, 12, 985-994. https://doi.org/10.2174/138527208785161187

2. Review: Jarosz, S.; Tiara, K.; Potopnyk, M.; Pure Appl. Chem., 2019, 97, 1137-1148. https://doi.org/10.1515/pac-2019-0116

3. Witkowski, G.; Jarosz, S. Synlett 2013, 1813-1817. https://doi.org/10.1055/s-0033-1339374

4. Magdycz, M.; Cmoch, P.; Jarosz, S. Heterocycles 2010, 80, 1303-1318. https://doi.org/10.3987/COM-09-S(S)120

5. Magdycz, M.; Jarosz, S. Tetrahedron: Asymmetry 2013, 24, 1412-1416. https://doi.org/10.1016/j.tetasy.2013.09.016

6. Evans, D. A.; Bartoli, J.; Shih, T. L. J. Am. Chem. Soc. 1981, 103, 2127-2129. https://doi.org/10.1021/ja00398a058

7. Evans, D. A.; Chapman, K. T.; Bisaha, J. Tetrahedron Lett. 1984, 25, 4071-4074. https://doi.org/10.1016/S0040-4039(01)90184-4

8. Evans, D. A.; Chapman, K. T.; Bisaha, J. J. Am. Chem. Soc. 1988, 110, 1238-1256. https://doi.org/10.1021/ja00212a037

9. Dransfield, P.J.; Moutel, S.; Shipman, M.; Sik, V. J. Chem. Soc., Perkin Trans. 1 1999, 3349-3355. https://doi.org/10.1039/a905706d

10. Witkowski, G.; Potopnyk, M. A.; Tiara, K.; Jarosz, S. Molecules 2020, 25, 3357. https://doi.org/10.3390/molecules25153357

11. Gaussian 09, Revision A.02, M. J. Frisch, G. W. Trucks, H. B. Schlegel, G. E. Scuseria, M. A. Robb, J. R. Cheeseman, G. Scalmani, V. Barone, G. A. Petersson, H. Nakatsuji, X. Li, M. Caricato, A. Marenich, J. Bloino, B. G. Janesko, R. Gomperts, B. Mennucci, H. P. Hratchian, J. V. Ortiz, A. F. Izmaylov, J. L. Sonnenberg, D. Williams-Young, F. Ding, F. Lipparini, F. Egidi, J. Goings, B. Peng, A. Petrone, T. Henderson, D. Ranasinghe, V. G. Zakrzewski, J. Gao, N. Rega, G. Zheng, W. Liang, M. Hada, M. Ehara, K. Toyota, R. Fukuda, J. Hasegawa, M. Ishida, T. Nakajima, Y. Honda, O. Kitao, H. Nakai, T. Vreven, K. Throssell, J. A. Montgomery, Jr., J. E. Peralta, F. Ogliaro, M. Bearpark, J. J. Heyd, E. Brothers, K. N. Kudin, V. N. Staroverov, T. Keith, R. Kobayashi, J. Normand, K. Raghavachari, A. Rendell, J. C. Burant, S. S. Iyengar, J. Tomasi, M. Cossi, J. M. Millam, M. Klene, C. Adamo, R. Cammi, J. W. Ochterski, R. L. Martin, K. Morokuma, O. Farkas, J. B. Foresman, and D. J. Fox, Gaussian, Inc., Wallingford CT, 2016. 